MS32-P09

\section{Combining crystal engineering strategies in the search for non-centrosymmetric single-crystal structures}

Jasper Van de Velde ${ }^{1}$, Frank Blockhuys ${ }^{1}$

1. University of Antwerp - Department of Chemistry, Antwerp, Belgium

email: jasper.vandevelde@uantwerpen.be

Despite the simplicity and robustness of inorganic NLO materials, their organic counterparts have been attracting attention due not only to their superior NLO performance but also to their faster electronic response and higher thermal stability [1]. The greatest advantage, though, at least from a chemical point of view, is the ability to design both the molecular and supramolecular structure in function of the desired properties or applications [2]. In the case of (lowest-order) single-component NLO materials, the main challenge consists of achieving non-centrosymmetry in the crystal lattice, which is a strict requirement to observe the desired optical effects.

Our previous work on organic optical materials focused on 1,4-distyrylbenzene $(1,4-\mathrm{DSB})$ derivatives with a wide variety of acceptor and donor substituents on the backbone. Such push-pull (A- $\pi$-D) compounds did yield non-centrosymmetric crystal structures, but the fraction of polar supramolecular structures remains low [3].

In order to maximize the probability for non-centrosymmetry, we now focus on 1,3-DSBs. This idea is supported by an analysis of the Cambridge Structural Database (CSD), which leads to the conclusion that compounds based on 1,3 -DSB crystallize non-centrosymmetrically in $25 \%$ of the cases, compared to $8 \%$ and $11 \%$ for $1,4-$ and $1,2-\mathrm{DSBs}$ respectively. Hence, for any system deviating enough from the typical rigid-rod shape of 1,4-DSBs, but not too much (1,2-DSBs), a higher probability for non-centrosymmetry can be expected. A large-scale quantum chemical screening of molecular 1,3-DSB based structures, in which hyperpolarizibilities, dipole moments and conformational energy differences were calculated, shows that next to A- $\pi-D$ all-carbon scaffolds, also D- $\pi$-D systems containing heterocyclic rings and nitrogen atoms in the spacers, can be of value not only in terms of NLO-properties but also in the light of the resulting supramolecular structure. As a result four crystal engineering strategies have been combined in an overall rational design: (1) the formation of an anti-parallel stacking should be hampered by the introduction of lateral steric hindrance between the constituent molecules (1,3DSBs), (2) the electronic ground-state dipole moment of the constituent molecules should be sufficiently small (D- $\pi-D)$, (3) the deviation from planarity of the constituent molecules should be increased (nitrogen-containing spacers) and (4) the most favorable conformation of the molecule in the light of strategies (1) and (2) should have an as high as possible energetic advantage with respect to other conformations.

A first series of 1,3-DSBs and 2,5-distyrylheteroarenes is crystallized and characterized with single-crystal X-ray diffraction in order to evaluate these strategies.
References:

[1] Reshak, A.H.; Shahimin, M.M.; Juhari, N. et al. (2013) Prog. Biophys. Mol. Biol. 113, 289-294.

[2] Dalton, L.R.; Harper, A.W.; Ghosn, R. et al. (1995) Chem. Matter. 7, 1060-1081.

[3] Collas, A.; De Borger, R.; Amanova, T. et al. (2011) New. J. Chem. 35, 649-662.

Keywords: nonlinear-optics, crystal engineering, polar crystals 